\title{
AVALIAÇÃO DO GPR PARA ESTIMATIVA DA PROFUNDIDADE DO SOLO EM UMA TRANSEÇÃO AFLORAMENTO DE QUARTZITOS-NEOSSOLO NO DF
}

\author{
Aldo Soares Pires Filho (aldopires1@hotmail.com - Graduando em Agronomia FAV-UNB); Tairone Paiva Leão \\ (tleao@unb.br - Docente FAV-UnB); Welitom Rodrigues Borges (welitom@unb.br - Docente IG-UnB); Isabela Resende \\ Almeida (isabellalmeida@hotmail.com - Graduanda em Geofísica IG-UnB); Márcio Maciel Cavalcanti \\ (marciom.cavalcanti@hotmail.com - Pós-graduando em Geociências Aplicadas IG-UnB)
}

Copyright 2012, SBGf - Sociedade Brasileira de Geofísica

Este texto foi preparado para a apresentação no V Simpósio Brasileiro de Geofísica Salvador, 27 a 29 de novembro de 2012. Seu conteúdo foi revisado pelo Comitê Técnico do V SimBGf, mas não necessariamente representa a opinião da SBGf ou de seus associados. É proibida a reprodução total ou parcial deste material para propósitos comerciais sem prévia autorização da SBGt.

\section{Resumo}

O uso de GPR para levantamento e prospecção de solos é recente, não havendo muitas aplicações em solos de condições tropicais. O objetivo deste trabalho foi o de avaliar o GPR para estimativa da espessura do solo em uma transeção afloramento de quartzitos-Neossolo Regolítico no DF. Os resultados foram comparados com tradagens, e constatou-se que as estimativas obtidas com o GPR foram muito próximas daquelas avaliadas pelo método convencional. Quando devidamente calibrado, o GPR é útil para a avaliação da profundidade de Neossolos Regolíticos arenosos.

\section{Introdução}

O solo é um dos recursos naturais mais importantes de um país, pois é dele que derivam os produtos para alimentar sua população. Nas regiões intertropicais, essa importância é maior ainda, por ser nessas regiões que se encontram a maioria dos países em desenvolvimento cuja economia depende de seus recursos naturais, especialmente as atividades agrícolas.

O solo passa gradualmente no seu limite inferior, em profundidade, para rocha dura ou materiais saprolíticos que não apresentam sinais de atividades animal, vegetal ou outras indicações da presença de atividade biológica (Embrapa, 2006). A determinação desses limites é fundamental para a agricultura, geotecnia e atividades urbanas. Porém, os métodos convencionais de estimativa de espessura de solo são demorados e onerosos, além do processo de coleta de dados não ser contínuo no espaço. Uma alternativa, rápida e de baixo custo, é o uso do método do radar de penetração no solo, conhecido mundialmente como Ground Penetrating Radar - GPR (Huisman et al., 2001). O GPR é utilizado há décadas nas áreas de geologia e geotecnia (Neal, 2004, Borges, 2007) sendo um método eletromagnético utilizado para localização de alvos subterrâneos rasos. O uso do GPR para levantamento de solos é relativamente recente (Huisman et al., 2001). O método GPR baseia-se na emissão e recepção de ondas eletromagnéticas através de antenas emissoras e receptoras, assim há o armazenamento dos dados em forma de traços que darão origem aos radargramas.

A utilização de GPR para levantamento de solos e determinação de conteúdo de água vem sendo observada na literatura internacional (Van Overmeeren et al., 1997; Van Dam, 2001; Huisman et al., 2003). Entretanto, no Brasil poucos são os estudos utilizando o GPR em pedologia (Ucha et al., 2002; Oliveira Junior \& Medeiros, 2008; Cezar et al., 2010; Cezar et al., 2011) e pouco se sabe sobre a adequabilidade e as limitações para aplicação de métodos eletromagnéticos em levantamentos de solos. É restrito também o conhecimento das interações entre a propagação de ondas eletromagnéticas e os minerais presentes nesses solos, as quais são distintas daqueles em solos de regiões temperadas, onde o método foi desenvolvido e testado.

Assim, este trabalho teve como objetivo avaliar a eficiência do GPR para estimativa da profundidade do solo em uma transeção afloramento de quartzitoNeossolo Regolítico.

\section{Metodologia/ Problema Investigado}

O trabalho foi realizado na Fazenda Água Limpa, campo experimental da Universidade de Brasília (Figura 1), a qual possui uma área de $42,36 \mathrm{~km}^{2}$, localizada na porção Sul-Sudoeste da região administrativa do Lago Sul, no DF, delimitada pelas coordenadas 47 $59^{\prime} 02,23^{\prime \prime} \mathrm{W}$ e $47^{\circ} 53^{\prime} 16,15^{\prime \prime} \mathrm{W}$ e $15^{\circ} 58^{\prime} 32,77^{\prime \prime} \mathrm{S}$ e $15^{\circ} 58^{\prime} 56,84^{\prime \prime} \mathrm{S}$ (Lacerda et al., 2007).

Os dados do GPR foram coletados em uma transeção de aproximadamente 572 metros (Figura 1). A transeção em estudo é formada por Neossolos Regolíticos compostos essencialmente de quartzo em fração areia derivados diretamente do intemperismo do quartzito, com afloramentos de rochas quartzíticas do Grupo Paranoá (Pádua et al., 2011). Foi utilizado para aquisição dos dados o sistema de offset único (Single Offset - SO) (Huisman et al., 2001). Para esse método foi utilizado um GPR SIR-3000 com antena blindada de $900 \mathrm{MHz}$. Ao longo da transeção avaliada com o GPR foram realizadas tradagens para comparação dos dados e assim verificar a eficiência do método em questão. 


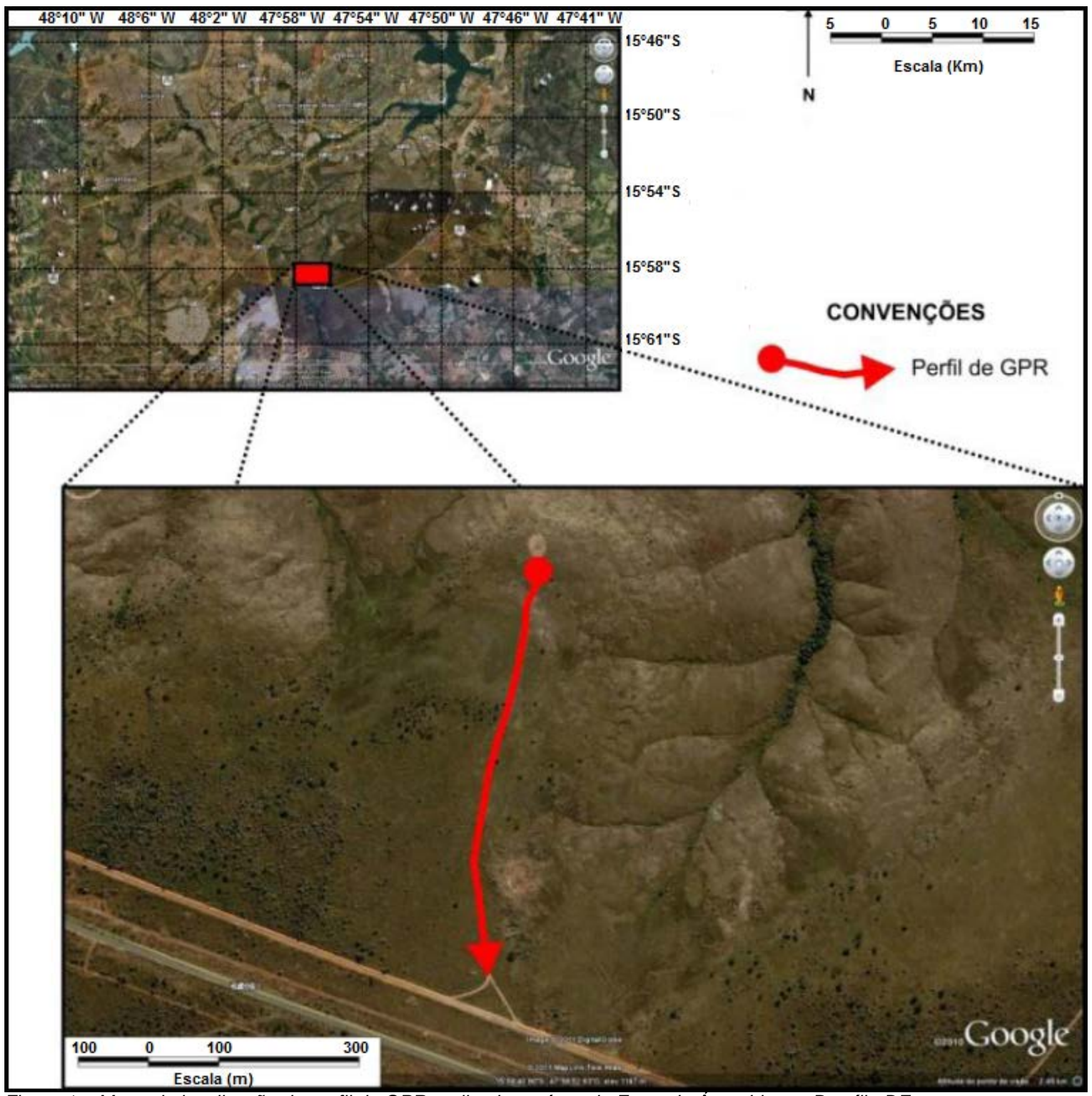

Figura 1 - Mapa de localização do perfil de GPR realizado na área da Fazenda Água Limpa, Brasília-DF.

Foi feita a correlação de Pearson e regressão linear entre as estimativas de profundidades do solo obtidas com o GPR e através de tradagem. Foi também feita uma interpretação das imagens de GPR, procurando identificar a profundidade do solo e a camada de rocha subjacente. Os radargramas foram também retirados para um perfil de Neossolo Regolítico aberto ao final da transeção de modo a validar a interpretação do sinal com os horizontes e camadas presentes no solo. As análises estatísticas foram realizadas no software SAS v9.2 e os dados geofísicos processados no Módulo 2D do software REFLEXW® versão 5.5.1 (Sandmeier, 2010). Durante o processamento foi determinado a chegada da primeira onda (ajuste do tempo zero). Após o processamento dos dados a estimativa da velocidade foi obtida através de informações geológicas. A profundidade de um refletor é determinada sendo substituída na equação (1) onde $h$ é a profundidade do refletor e $t$ o tempo que a onda demorou para ser emitida, refletida e captada, foi obtida uma 
profundidade através do trado, para que com essa fosse calibrado todo o perfil do GPR.

$v=\frac{2 h}{t}$

\section{Resultados}

A correlação de Pearson ( $r$ ) entre a profundidade do solo medida por meio de tradagem $\left(P_{\text {trado }}\right)$ e aquela estimada pelo GPR $\left(P_{G P R}\right)$ foi de $0,99(P>r<0.0001)$ indicando que o GPR foi preciso em estimar a profundidade do solo (Figura 2). $O r^{2}$ para a equação de regressão entre $P_{\text {trado }}$ e $P_{G P R}$ foi de 0,99 com coeficiente angular próximo a 1 e o intercepto não foi estatisticamente diferente de 0 e portanto não foi incluso na equação.

Um perfil característico de Neossolo Regolítico encontrado na área pode ser visualizado na Figura 3. Este solo em particular possui uma camada de detritos de quartzito e quartzo iniciando a uma profundidade de aproximadamente 30 a $40 \mathrm{~cm}$ sobrejacente a uma camada de saprolito de quartzito iniciando a aproximadamente $79 \mathrm{~cm}$. No radargrama da Figura 3 observa-se que o GPR foi eficiente em distinguir a camada detrítica, sendo assim possível diferenciar o horizonte superficial acima e o saprolito abaixo. Neste solo a camada detrítica e o saprolito constituem impedimento físico a penetração de raízes e infiltração livre de água e assim a profundidade do solo efetiva estimada pelo GPR ao longo da transeção deve corresponder ao topo da camada detrítica. A transeção completa pode ser observada na Figura 4. A linha em preto na imagem do GPR marca a profundidade do solo estimada pelo GPR, enquanto a profundidade estimada, em $m$, pode ser vista na imagem abaixo (com exagero vertical). A margem esquerda da imagem corresponde ao afloramento de quartzito, na borda da segunda superfície geomorfológica, enquanto pode ser visto um aprofundamento do solo em direção a margem direita correspondente a um relevo mais plano e acúmulo de solo residual. Nos locais mais profundos o material de solo superficial é formado predominantemente de fração areia composta de quartzo acima da camada detrítica e do saprolito.

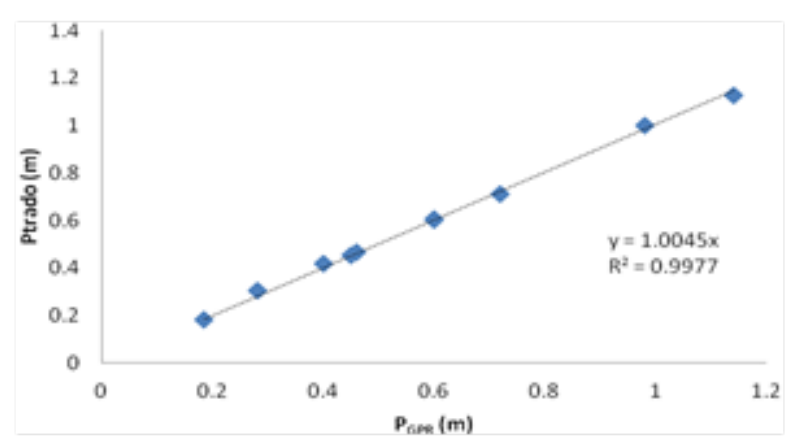

Figura 2 - Gráfico de dispersão entre valores de profundidade medidos com o trado e estimados com GPR.

\section{Conclusões}

O GPR mostrou-se um método rápido e preciso para estimativa da profundidade de um Neossolo Regolítico quando comparado com o método da tradagem. Outro aspecto positivo na utilização do GPR é que esse proporciona uma aquisição de dados contínua diferentemente dos métodos comumente utilizados onde há um intervalo de espaço entre uma medida e a outra, havendo necessidade de interpolação entre as medidas, o que insere incerteza no processo.

\section{Agradecimentos}

A direção da Fazenda Água Limpa pela permissão para realização dos experimentos. Ao Instituto de Geociências e Laboratório de Geofísica Rasa da UnB pelo apoio logístico, operacional e equipamentos. A Divisão Técnico Científica do Instituto Nacional de Criminalística da Polícia Federal do Distrito Federal pelo empréstimo da roda odométrica do GPR.

\section{Referências}

Borges, W. R., 2007. Caracterização geofísica de alvos rasos com aplicações no planejamento urbano e do meio ambiente: Estudo sobre o sítio controlado do IAG/USP. Tese de Doutorado: IAG/USP. 271p

Cezar, E., Nanni, M. R., Chicati, M. L., Fabrio, F. D. Hata, F. T. \& Oliveira, R. B., 2010. Uso de sistema GPR (Ground Penetrating Radar) na avaliação de atributos de um solo sob plantio de cana-de-açúcar. Revista Brasileira de Ciência de Solo, 34:291-297.

Cezar, E., Nanni, R. M., Chicati, L. M. \& Oliveira, R. B., 2011. Emprego de GPR no estudo de solos e sua correlação com métodos laboratoriais. Anais $\mathrm{XV}$ Simpósio Brasileiro de Sensoriamento Remoto, Curitiba, Brasil, p. 9112-9119.

EMBRAPA, 2006. Sistema brasileiro de classificação de solos. Embrapa Solos. 306p

Huisman, J., Hubbard, S. S., Redman, J. D. \& Annan, A. P., 2001. Measuring soil water content with ground penetrating radar: a review. Vadose Zone Journal, 2:476491

Huisman, J. A., Hubbard S. S., Redman J. D. \& Annan A. P. 2003. Measuring Soil Water Content with Ground Penetrating Radar: A Review. Vadose Zone Journal, 2: 476-491.

Lacerda, M. P. C., Barbosa, I. O., Campos, P. M. \& Papa, R. A., 2007. Utilização de sensoriamento remoto para o 
estabelecimento de relações entre vegetação nativa e classes de solos em mapeamento pedológico, Distrito Federal. Anais XIII Simpósio Brasileiro de Sensoriamento Remoto, Florianópolis, Brasil, 21-26 abril 2007, INPE, p. 3991-3996

Neal, A., 2004. Ground-penetrating radar and its use in sedimentology: principles problems and progress. EarthScience Reviews, 66:261-330

Oliveira Junior, J. G. O. \& Medeiros, W. E. 2008. Influência do conteúdo de água, granulometria e minerais pesados sobre as reflexões de GPR em corpos arenosos inconsolidados. Revista Brasileira de Geofísica, 26:437449.

Pádua, A. I., Borges, W. R., Cunha, L. S., Leão, T. P. \& Rocha, S. R., 2011. Estimativa da velocidade eletromagnética do GPR através do mergulho verdadeiro de camadas de Quartzito do grupo Paranoá, Distrito Federal. In: International Congress of the Brazilian Geophysical Society \& EXPOGEF, 12, Rio de Janeiro-RJ

Sandmeier, K. J., 2010. REFLEXW Version 5.5, Windows $9 \times / 2000 / N T / X P / V I S T A / 7$. Program for the processing of seismic, acoustic or electromagnetic reflection, refraction and transmission data. Manual do Software, Karlsruhe, Germany, 209p.

Ucha, J. M., Botelho, M., Vilas Boas, G. S., Ribeiro, L. P. \& Santana, P. S., 2002. Uso do radar penetrante no solo (GPR) na investigação dos solos dos tabuleiros costeiros no litoral Norte do estado da Bahia. Revista Brasileira de Ciência do Solo, 26:373-380.

Van Dam, R. L., 2001. Causes of ground-penetrating radar reflections in sediment. Doctoral Thesis - Faculty of Earth Sciences, Vrije Universiteit, The Netherlands, 110p.

Van Overmeem R. A., Sariowan S. V. \& Gehrels J. C., 1997. Ground penetrating radar for determining volumetric soil water content; results of comparative measurements at two test sites. Journal of Hydrology, 197:316-338. 

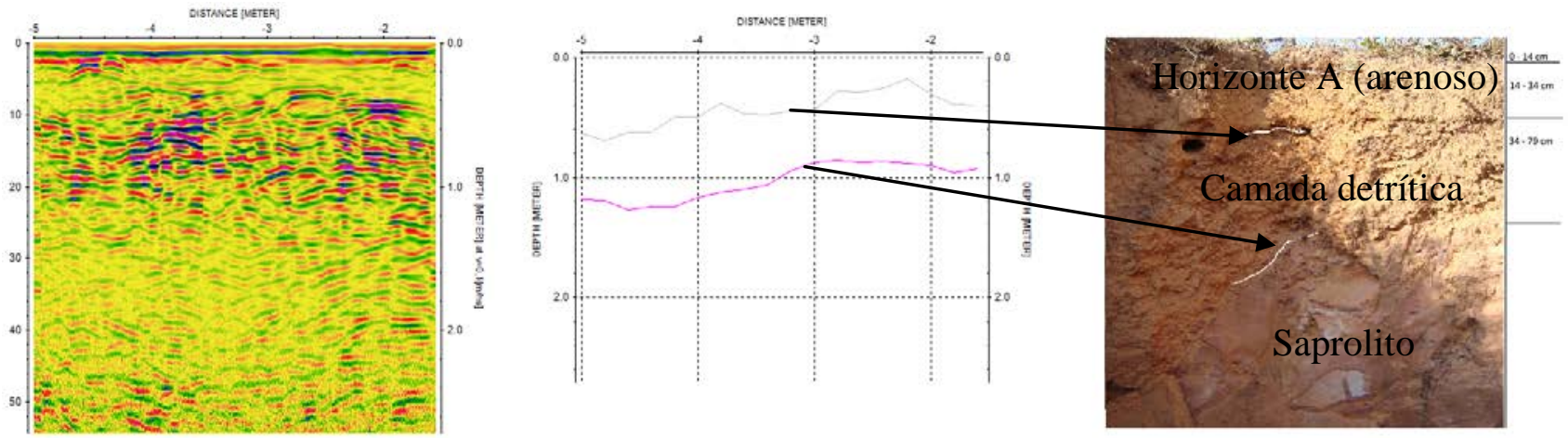

Figura 3 - Imagem adquirida utilizando o GPR, profundidade estimada e fotografia de perfil correspondente de Neossolo Regolítico avaliado no estudo.

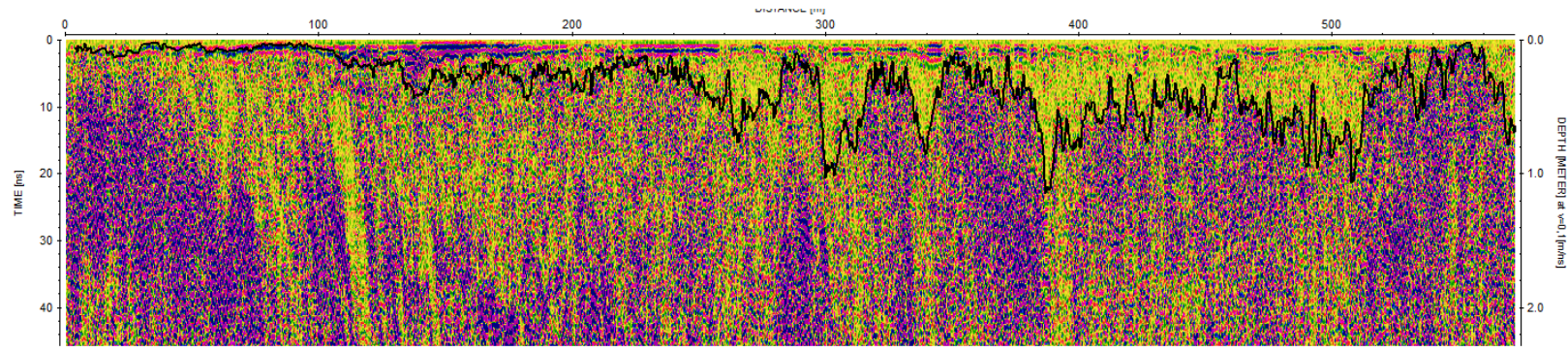

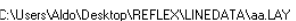

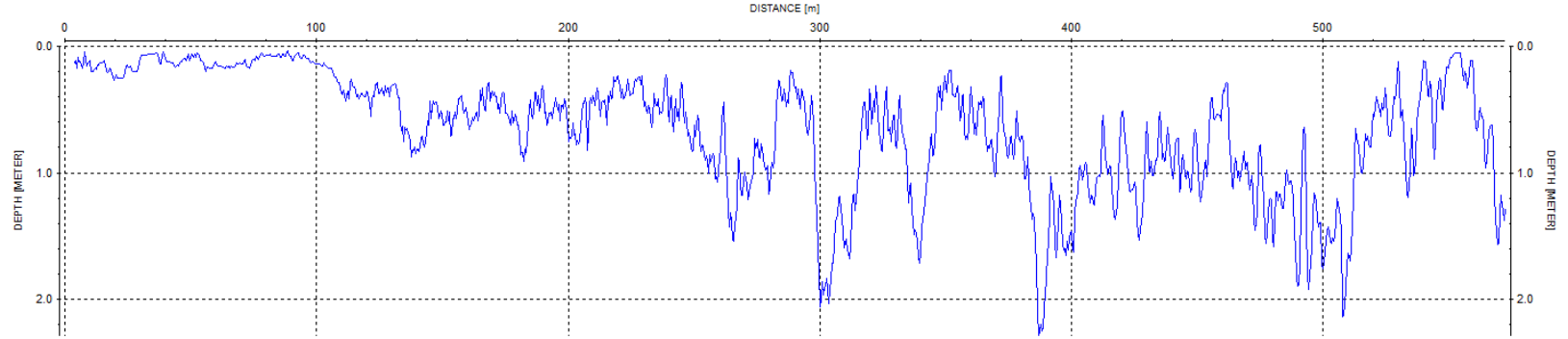

Figura 4 -. Transeção completa adquirida para avaliação da profundidade do solo utilizando GPR (acima) e profundidade estimada (abaixo). 